cells (HSPCs). 57th Am Soc Hematol Ann Meet Expo, Orlando, FL, USA, 4-8 December 2015 (abstract 4301).

This work is licensed under a Creative Commons AttributionNy NC SA NonCommercial-ShareAlike 4.0 International License. The images or
other third party material in this article are included in the article's Creative Commons license, unless indicated otherwise in the credit line; if the material is not included under the Creative Commons license, users will need to obtain permission from the license holder to reproduce the material. To view a copy of this license, visit http:// creativecommons.org/licenses/by-nc-sa/4.0/

(c) The Author(s) 2016

Supplementary Information accompanies this paper on the Leukemia website (http://www.nature.com/leu)

\title{
OPEN
}

\section{Evidence of shared epitopic reactivity among independent B-cell clones in chronic lymphocytic leukemia patients}

Leukemia (2016) 30, 2419-2422; doi:10.1038/leu.2016.245

Chronic lymphocytic leukemia (CLL) is a neoplastic disease of mature B cells that express a functional immunoglobulin (lg) B-cell receptor (Ig-BCR) on the cell surface. ${ }^{\prime}$ The Ig-BCR includes the binding site (idiotype) for the epitope of cognate antigen, which results from the stochastic and productive lg variable genes rearrangement and possible somatic hypermutation. Individual $\mathrm{B}$-cell clones differ from one another because of the diverse amino acid sequences within the idiotype of the expressed $\mathrm{Ig}-\mathrm{BCR}^{2}$ so that the epitopic specificity is shared in a given clonal B-cell population.

Several studies have recently highlighted two peculiar characteristics that distinguish CLL among other mature B-cell malignancies. First, the $\mathrm{Ig}-\mathrm{BCR}$ repertoire of CLL is biased, as it is restricted toward the preferential usage of certain Ig heavy- $\left(\lg _{\mathrm{H}}\right)$ and light $\left(\lg V_{K} / \lambda\right)$-chain variable genes, ${ }^{3,4}$ and unusually similar, stereotyped heavy-chain complementarity determining region 3 $\left(\mathrm{V}_{\mathrm{H}} \mathrm{CDR} 3\right)$ amino acid sequences. ${ }^{5}$ The skewed Ig-BCR could be owing to an Ig-BCR-driven selection mechanism initiated by specific antigens that promote the expansion and possibly the maintenance of the cognate CLL clone. ${ }^{6}$ Consistent with this hypothesis, several studies have demonstrated the reactivity of CLL Ig-BCR against foreign antigens, self-antigens, peptides and intrinsic $\operatorname{lgV}_{\mathrm{H}}$ motif. ${ }^{7}$ Second, $\mathrm{CLL}$ is not always a monoclonal disorder, as two or multiple CLL clones have been found in $2-5 \%$ of CLL patients. ${ }^{8,9}$ Furthermore, the monoclonal B-lymphocytosis precursor state, which precedes the clinically relevant leukemic phase in virtually all CLL patients, sometimes involves multiple B-cell clones. ${ }^{10}$

It is still unknown whether two or more CLL clonotypes within the same patient potentially associate with the same antigenic reactivity, or alternatively arise as stochastic and antigenindependent events, fostered by the accumulation of oncogenic abnormalities in the preleukemic state. To answer this question, here we have characterized the epitope recognition profiles of CLL clonotypes by coupling the genetic analysis of Ig variable genes and the epitopic reactivity at single-cell level.

We isolated single $\mathrm{CD}^{+} \mathrm{B}$ cells from peripheral blood of six newly diagnosed untreated CLL patients, randomly referred to the Medical Oncology Unit-University Magna Graecia of Catanzaro. CLL patients displayed the typical CLL immunophenotype, with no evidence of separate/aberrant B-cell populations (Supplementary Table S1). We determined the $V_{H} D J_{H}$ and $V_{L} J_{L}$ complementary DNA (CDNA) sequence of at least 20 single leukemic cells per patient (Supplementary Table S2). All cDNA sequences showed a productive rearrangement at the heavy- and light-gene loci. In particular, we detected single and distinct $V_{H} D J_{H}$ and $V_{L} D_{L}$ rearrangements in CLL\#1, CLL\#2 and CLL\#3, indicating the presence of a single clonotype (Table 1, Supplementary Table S2). Differently, CLL\#4, CLL\#5 and CLL\#6 exhibited two different $\mathrm{V}_{\mathrm{H}} \mathrm{DJ} \mathrm{J}_{\mathrm{H}}$ rearrangements, each of them pairing with a unique and

Table 1. Characteristics of CLL clonotypes and mimotopes

\begin{tabular}{|c|c|c|c|c|c|c|c|c|c|c|c|}
\hline Patient & Clonotype & $m l g C L L^{\mathrm{a}}$ & $V_{H} D J_{H}$ & $V_{L} J_{L}$ & $\begin{array}{l}\text { CDR3 } \\
\text { length }^{\mathrm{b}}\end{array}$ & VH CDR3 IMGT aa sequence ${ }^{c}$ & $\begin{array}{l}\text { Freq. } \\
(\%)^{\mathrm{d}}\end{array}$ & $\begin{array}{l}\text { Stereotypic } \\
\text { subset }^{\mathrm{e}}\end{array}$ & $\begin{array}{l}\text { Mimotope } \\
\text { name }\end{array}$ & $\begin{array}{l}\text { Mimotope aa } \\
\text { sequence }\end{array}$ & $\underset{(n M)^{f}}{\mathrm{~K}_{D^{f}}}$ \\
\hline CLL\#1 & CLL1 & mlgCLL1 & V3-11/D3-10/J6 & $\kappa V 1-39(1 D-39) / J 2$ & 17 & AREGLWFGELSYYGMDV & 100 & NA & pCLL1 & CSPAKELGC & 34.03 \\
\hline \multirow[t]{2}{*}{ CLL\#2 } & CLL2 & mlgCLL2-1 & V3-73/D3-3/J6 & $\kappa \mathrm{V} 1-33(1 \mathrm{D}-33) / \mathrm{J}$ & 20 & TFDFWSGYYDGYYYYYGMDV & 80 & NA & pCLL2-1 & CNTYSVSLC & 10.81 \\
\hline & & mlgCLL2-2 & & & & TFDFWSGYYDGYYYYYGLDF & 20 & & pCLL2-2 & CKSYSVSLC & 7.57 \\
\hline \multirow[t]{2}{*}{ CLL\#3 } & CLL3 & mlgCLL3-1 & V3-23/D3-22/J4 & $\kappa V 1-8(1 D-8) J 3$ & 17 & AKRDYSHRSDYAPLFEY & 90 & NA & pCLL3-1 & CPPQSVTEC & 22.14 \\
\hline & & mlgCLL3-2 & & & & GKRDYSHSSDYAPLFEY & 10 & NA & pCLL3-2 & CDVWHSAYC & 5.64 \\
\hline CLL\#4-1 & CLL4-1 & mlgCLL4-1 & V4-34/D7-27/J2 & кV1-33(1D-33)/J1 & 17 & ARRGTGDPPYWYFDL & 77 & NA & pCLL4-1 & CTTNPADSC & 5.50 \\
\hline CLL\#4-2 & CLL4-2 & mlgCLL4-2 & V4-4/D6-19/J2 & $\kappa V 2-28(2 D-28) J 1$ & 21 & ARGTVGQQWLEVLDWYFGL & 23 & NA & pCLL4-2 & CVLWWSPIC & 3.90 \\
\hline CLL\#5-1 & CLL5-1 & mlgCLL5-1 & V4-34/D3-22/J4 & KV1D-12/J1 & 20 & ARGGNNDKIVMLLYYFDF & 57 & NA & pCLL5-1 & CFSDDEWWC & 7.08 \\
\hline CLL\#5-2 & CLL5-2 & mlgCLL5-2 & V4-59/D3-22/J3 & $\kappa V 1-13(1 D-13) / J 2$ & 18 & ARDYDYDTRKSDAFDIW & 43 & NA & pCLL5-2 & CPPFTNYEC & 7.32 \\
\hline CLL\#6-1 & CLL6-1 & mlgCLL6-1 & $\mathrm{V} 1-46 / \mathrm{D} 3-10 / \mathrm{J} 6$ & $\kappa V 3 D-15 / J 1$ & 22 & ARDWVATMVRGVIESRPTGMDV & 65 & NA & pCLL6-1 & CNQDWHKKC & 57.53 \\
\hline CLL\#6-2 & CLL6-2 & mlgCLL6-2 & V4-34/D2-2/J6 & $\kappa V 1 D-17 / J 1$ & 24 & ASRFYCSGSSCEAPRYYYYYGLDV & 35 & 16 & pCLL6-2 & CTTVIPERC & 22.67 \\
\hline
\end{tabular}

${ }^{a}$ Name of the CLL-derived monoclonal lg $\gamma 1$ antibodies expressing the indicated $V_{H} D J_{H}$ and $V_{L} J_{L}$ rearrangements. ${ }^{b} \mathrm{Number}$ of amino acids of the $\mathrm{VH}_{\mathrm{C}} \mathrm{CDR}$. ${ }^{\mathrm{C}} \mathrm{VH}$ CDR3 amino acid sequence according to the International ImMunoGeneTics information system (http://www.imgt.org). ${ }^{d}$ Percentage of analyzed cells expressing the indicated $\mathrm{VH}$ CDR3. ${ }^{\text {e}}$ Stereotypic subset according to Agathangelidis et $a l^{5}{ }^{5} \mathrm{NA}$, not attributable to a currently defined stereotypic subset. ${ }^{\mathrm{f}} K_{\mathrm{D}}$ values for the mimotope binding to the cognate $\mathrm{mlgCLL}$, as estimated by the Scatchard plot analysis. 
distinct $V_{L} J_{L}$ rearrangement, indicating the coexistence of two clonotypes (Table 1, Supplementary Table S2). The coexistence of two clonotypes in CLL\#4, CLL\#5 and CLL\#6 was confirmed by the heteroduplex analysis and sequencing of $V_{H} D J_{H}$ and $V_{L} J_{L}$ rearrangements amplified from the bulk CLL cells (Supplementary Figure S1). Of note, the VK usage of clonotypes CLL5-1, CLL6-1 and CLL6-2 were univocally assigned to $V_{K}$ genes of the distal cluster, which is evocative of receptor editing.

The analysis of nucleotide differences among the $V_{H}$ sequences of the same clonotype showed a variable degree of base-pair substitutions (Supplementary Table S3). Despite the intraclonal diversity of the $\mathrm{V}_{\mathrm{H}}$ region, each clonotype showed either an identical or two quasi-identical $\mathrm{V}_{\mathrm{H}}$ CDR3 amino acid sequences (Table 1 and Supplementary Table S3). By comparing the $V_{H}$ CDR3 amino acid sequence of clonotypes with a data set of stereotypic Ig-BCRs, ${ }^{5}$ CLL6-2 was found to be a member of the major subset 16 , whereas other clonotypes did not fall within the stereotypic subsets (Table 1).

Next, we aimed to identify peptide mimetics of the natural epitope (hereafter, referred as mimotopes) that bound to the lg-BCR of CLL clonotypes. To this end, we produced monoclonal antibodies IgG1 (mlgCLLs) that carried the $V_{H} D J_{H}$ and $V_{L} J_{L}$ rearrangements of $C L L$ clonotypes linked to an $\lg H C \gamma 1$, or $\lg L C_{K} / \lambda$ segment, as described ${ }^{11}$ (Table 1, Supplementary Figure S2). The

a

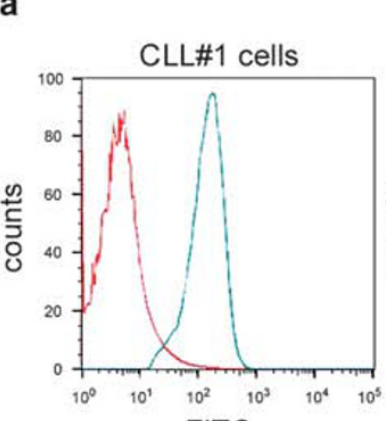

FITC

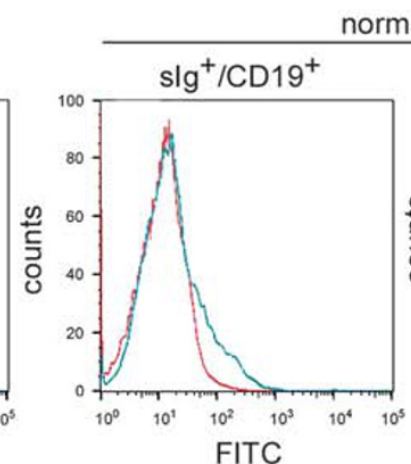

FITC

normal PBMCs

b

$$
\begin{gathered}
\text { biotin-pCLL1 } \\
+ \\
\text { strept-FITC }
\end{gathered}
$$
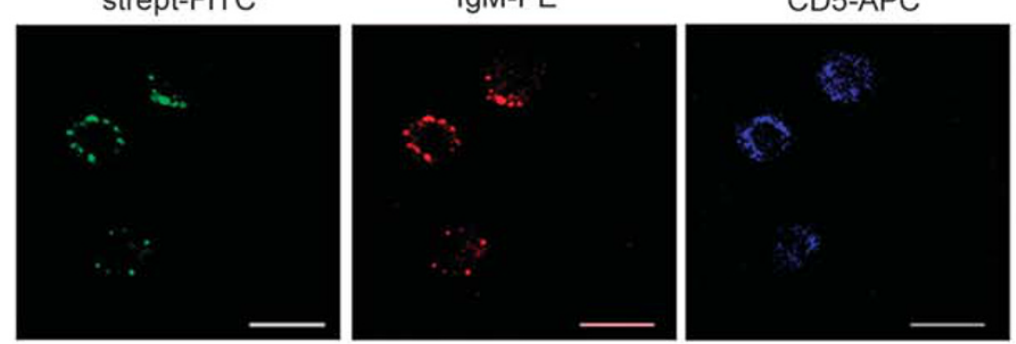

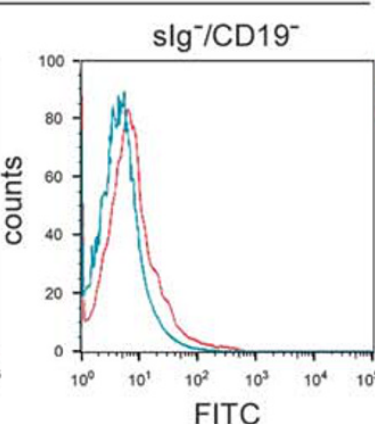

FITC

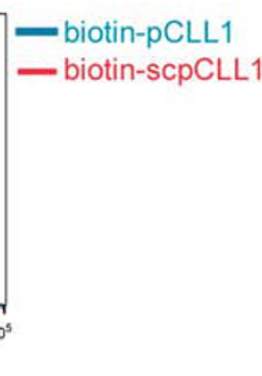

\begin{tabular}{|c|c|c|c|c|c|c|c|c|c|c|c|}
\hline & mlgCLL1 & mlgCLL2-1 & $\mathrm{mlgCLL2-2}$ & mlgCLL3-1 & mlgCLL3-2 & mlgCLL4-1 & mlgCLL4-2 & mlgCLL5-1 & mlgCLL5-2 & mlgCLL6-1 & mlgCLL6-2 \\
\hline pCLL1 & & & & & & & & & & & \\
\hline pCLL2-1 & & & & & & & & & & & \\
\hline pCLL2-2 & & & & & & & & & & & \\
\hline pCLLL3-1 & & & & & & & & & & & \\
\hline pCLLL3-2 & & & & & & & & & & & \\
\hline pCLLL4-1 & & & & & & & & & & & \\
\hline pCLL4-2 & & & & & & & & & & & \\
\hline pCLL5-1 & & & & & & & & & & & \\
\hline pCLLL-2 & & & & & & & & & & & \\
\hline pCLL6-1 & & & & & & & & & & & \\
\hline pCLL6-2 & & & & & & & & & & & \\
\hline
\end{tabular}

C

ELISA Absorbance. Fold relative to blank: $>8$ fold $5<$ fold $<8 \quad 3<$ fold $<5 \quad 2<$ fold $<3 \quad<2$ fold

Figure 1. Mimotope-binding profile of CLL. (a) Specificity of mimotope binding to cognate CLL cells by flow cytometric analysis. Non-permeabilized $\mathrm{CD}^{+}$cells $\left(1 \times 10^{6}\right.$ ) from CLL\#1 patient (left panel), or $\mathrm{slg}^{+} / \mathrm{CD} 19^{+}$(middle panel) and $\mathrm{slg}^{-} / \mathrm{CD} 19^{-}$(right panel) peripheral blood mononuclear cells (PBMCs) $\left(1 \times 10^{6}\right.$ ) from a pool of healthy donors were incubated with $\mathrm{N}$-biotinylated pCLL1 mimotope (10 pM), or control $\mathrm{N}$-biotinylated scrambled peptide. Peptide binding to CLL cells was revealed by streptavidin-FITC. (b) Colocalization of $\mathrm{N}$-biotinylated pCLL1 mimotope with the surface IgBCR of CLL\#1 cells. CD5 $5^{+}$cells $\left(1 \times 10^{6}\right)$ from CLL\#1 patient (left panel) were incubated with $N$-biotinylated pCLL1 mimotope (10 pM), stained with strepavidin-FITC (1:100), anti-human-IgM PE (1:100), anti-CD5-APC (1:100) and analyzed by confocal microscopy. Scale bar: $5 \mu$ m. (c) Enzyme-linked immunosorbent assay (ELISA)-binding reactivity of selected mimotopes against the mlgCLLs. ' $\mathrm{pCLL}$ ' is the labeling for peptide (mimotope) ligand; ' $\mathrm{mlgCLL}$ ' is the labeling for the recombinant, CLL-derived lg. Relative absorbance was calculated as the difference between optical density $405 \mathrm{~nm}$ and optical density $620 \mathrm{~nm}$ divided by the absorbance of the blank sample, and reported as shades of green at the bottom. The white square indicates ELISA absorbance value less than twofold relative to blank, indicating no interaction in ELISA. 
mlgCLLs were used as baits for screening a constrained (CX7C) random phage-displayed peptide library, ${ }^{12}$ which resulted in the isolation of at least 25 phage clones for each mlgCLL bait (Supplementary Table S4). We observed a considerable difference of enzyme-linked immunosorbent assay (ELISA)-binding reactivity among the phage clones isolated through the same $\mathrm{mlgCLL}$ (Supplementary Figure S3). As mimotopes are equally distributed among phage particle (five copies per phage), we assumed that the differences in the binding affinity of distinct mimotopes to the same mlgCLL might account for the differences in the ELISAbinding reactivity.

Hence, we synthesized a number of peptides corresponding to the phage-displayed mimotopes that had shown the highest binding affinity toward the cognate $\mathrm{mlgCLL}$ (Table 1). Synthetic mimotopes bound in a dose-dependent manner to the cognate mlgCLL, whereas tested negative for control human polyclonal Igs (Supplementary Figure S4), demonstrating that the selected mimotopes maintained the mlgCLL-binding ability out of the phage context. The equilibrium constant $K_{\mathrm{D}}$ of synthetic mimotope binding to the cognate mlgCLL was in the nanomolar range, as estimated by the Scatchard plot analysis (Table 1).

All mimotopes bound to the corresponding CLL cells, and not to normal $B\left(\mathrm{slg}^{+} / \mathrm{CD}_{19}{ }^{+}\right)$or non-B cells $\left(\mathrm{slgG}^{-} / \mathrm{CD} 19^{-}\right)$isolated from the peripheral blood of a pool of healthy donors, as shown by the fuorescence-activated cell sorting analysis (Figure 1a). The mimotopes binding to B-CLL cells was competed in a dosedependent manner by soluble cognate mlgCLLs and not by polyclonal human lgG1 (Supplementary Figure S5). Moreover, mimotopes colocalized with the slg-BCR on the surface of the cognate B-CLLs, as shown by the confocal microscopy (Figure 1b). Overall, these data confirmed that the mimotope binding to the corresponding B-CLL cells was specifically achieved through direct interaction with the slg-BCR, thus validating the selected mimotopes as truly representative of the epitopic reactivity of the original CLL cells.

Then, we performed a systematic characterization of the epitopic reactivity of CLL clonotypes by probing the binding of individual mlgCLLs to all selected mimotopes by ELISA. Each mlgCLL was able to bind not only to its cognate mimotope, but also to distinct mimotopes of others mlgCLLs (Figure 1c). These results highlighted a certain degree of common epitopic reactivity among the mlgCLLs, as previously reported by others. $^{13,14}$

A first level of common reactivity regarded the mlgCLLs derived from the same patient that invariably shared the epitopic reactivity, regardless they were classified as distinct clonotypes. In particular, the mlgCLLs with quasi-identical $V_{H} C D R 3$, which were derived from patients harboring a unique clonotype (mlgCLL2-1 and mlgCLL2-2 from patient CLL\#2; mlgCLL3-1 and mlgCLL3-2 from patient CLL\#3), equally bound to the two selected mimotopes (pCLL2-1 and pCLL2-2 for patient CLL\#2; pCLL3-1 and pCLL3-2 for patient CLL\#3), thus indicating that the small differences in their $V_{H}$ CDR3 did not affect their binding affinity (Figure 1c). The mlgCLLs derived from patients harboring two different clonotypes (mlgCLL4-1 and mlgCLL4-2; mlgCLL5-1 and mlgCLL5-2; mlgCLL6-1 and mlgCLL6-2) also showed common epitopic reactivity, even though with different ELISA reactivity. Thus, the diverse $V_{H}$ CDR3 pattern of distinct CLL clonotypes harbored by the same patient did not prevent the binding to a common set of mimotopes.

A second level of shared epitopic reactivity was observed for some mlgCLLs derived from different patients. In particular, the mlgCLLs derived from patients CLL\#1, CLL\#2 and CLL\#6 recognized the same subset of mimotopes (pCLL1, pCLL2-1, pCLL2-2, pCLL6-1 and pCLL6-2; Figure 1c). The mlgCLL4-2 also shared a similar ELISA-binding profile, except for the binding to pCLL1-2.

The clonotypic mlgCLL1, mlgCLL2, mlgCLL4-2, mlgCLL6-1 and mlgCLL6-2 did not show any obvious similarity in the $V_{H}$ CDR3 primary structure, or a preferential usage of $V_{H}$ and $V_{L}$ regions that would account for the common binding to the same subset of mimotopes. Hence, their shared epitopic reactivity should lie on structural motifs of the whole $\mathrm{V}_{\mathrm{H}}$ and $\mathrm{V}_{\mathrm{L}}$ regions, which will require further investigation.

Overall, these data demonstrated that (a) mlgCLLs derived from independent B-cell clones of the same CLL patient shared the epitopic reactivity and (b) a subset of CLLs exhibited some degree of common epitope reactivity.

Our results confirmed recent findings on the appearance of two or more independent B-CLL clones within the same patient. ${ }^{8-10}$ Importantly, we document for the first time that the Ig-BCRs of unrelated clonotypes in the same CLL patient may recognize common epitopes, thus revealing a striking homogeneity, grounded on epitopic reactivity, that was unpredictable from the molecular features of the Ig-BCR, including the CDR3 composition and length. The coexistence within the same patient of two unrelated CLLs clonotypes associated with the same epitopic reactivity reinforces the key role of antigenic selection in CLL pathogenesis and demand a more exhaustive understanding of the biology of this disease based on epitopic reactivity.

\section{CONFLICT OF INTEREST}

The authors declare no conflict of interest.

\section{ACKNOWLEDGEMENTS}

This work was supported by Ministero della Salute Grants GR-2009-1606801 (to CP) and RF-2010-2306943 (to GS), and Ministero dell'Istruzione, dell'Università e della Ricerca Grant POR CALABRIA FSE 2007/2013 (to GS), International Agency for Research on Cancer (AIRC) Grant IG-2009- 9411 (to GS), and COFIN-MIUR (to GS and IQ). We thank Dr Michel Nussenzweig (The Rockefeller University Hospital, NY, USA) for plasmids expressing the $\lg \gamma 1$, $\lg k$ or $\lg \lambda$ constant regions.

\section{S Mimmi ${ }^{1,3}$, E Vecchio $^{1,3}$, E laccino $^{1}$, M Rossi $^{1}$, A Lupia $^{1}, \mathrm{~F}$ Albano ${ }^{1}$, F Chiurazzi $^{2}$, G Fiume $^{1}$, A Pisano ${ }^{1}$, S Ceglia ${ }^{1}$, M Pontoriero ${ }^{1}$ G Golino ${ }^{1}$, P Tassone ${ }^{1}$, I Quinto ${ }^{1}$, G Scala ${ }^{1,3}$ and C Palmieri, ${ }^{1,3}$ \\ ${ }^{1}$ Department of Experimental and Clinical Medicine, University 'Magna Graecia' of Catanzaro, Catanzaro, Italy and ${ }^{2}$ Department of Clinical Medicine, University 'Federico II' of Naples, Naples, Italy E-mail: quinto@unicz.it or cpalmieri@unicz.it ${ }^{3}$ These authors contributed equally to this work.}

\section{REFERENCES}

1 Zenz T, Mertens D, Kuppers R, Dohner H, Stilgenbauer S. From pathogenesis to treatment of chronic lymphocytic leukaemia. Nat Rev Cancer 2010; 10: 37-50.

2 Hershberg U, Luning Prak ET. The analysis of clonal expansions in normal and autoimmune B cell repertoires. Philos Trans R Soc Lond B Biol Sci 2015; 370: 1-16.

3 Ghiotto F, Fais F, Valetto A, Albesiano E, Hashimoto S, Dono M et al. Remarkably similar antigen receptors among a subset of patients with chronic lymphocytic leukemia. J Clin Invest 2004; 113: 1008-1016.

4 Messmer BT, Albesiano E, Efremov DG, Ghiotto F, Allen SL, Kolitz J et al. Multiple distinct sets of stereotyped antigen receptors indicate a role for antigen in promoting chronic lymphocytic leukemia. J Exp Med 2004; 200: 519-525.

5 Agathangelidis A, Darzentas N, Hadzidimitriou A, Brochet X, Murray F, Yan XJ et al. Stereotyped B-cell receptors in one-third of chronic lymphocytic leukemia: a molecular classification with implications for targeted therapies. Blood 2012; 119: 4467-4475.

6 Stevenson FK, Krysov S, Davies AJ, Steele AJ, Packham G. B-cell receptor signaling in chronic lymphocytic leukemia. Blood 2011; 118: 4313-4320.

7 Sutton LA, Rosenquist R. The complex interplay between cell-intrinsic and cellextrinsic factors driving the evolution of chronic lymphocytic leukemia. Semin Cancer Biol 2015; 34: 22-35.

8 Plevova K, Francova HS, Burckova K, Brychtova Y, Doubek M, Pavlova S et al. Multiple productive immunoglobulin heavy chain gene rearrangements in 
chronic lymphocytic leukemia are mostly derived from independent clones. Haematologica 2014; 99: 329-338.

9 Sanchez ML, Almeida J, Gonzalez D, Gonzalez M, Garcia-Marcos MA, Balanzategui $\mathrm{A}$ et al. Incidence and clinicobiologic characteristics of leukemic B-cell chronic lymphoproliferative disorders with more than one B-cell clone. Blood 2003; 102: 2994-3002.

10 Klinger M, Zheng J, Elenitoba-Johnson KS, Perkins SL, Faham M, Bahler DW. Nextgeneration IgVH sequencing CLL-like monoclonal B-cell lymphocytosis reveals frequent oligoclonality and ongoing hypermutation. Leukemia 2015; 30: 1055-1061.

11 Tiller T, Meffre E, Yurasov S, Tsuiji M, Nussenzweig MC, Wardemann $H$. Efficient generation of monoclonal antibodies from single human B cells by single cell RT-PCR and expression vector cloning. J Immunol Methods 2008; 329: $112-124$

12 Palmieri C, Falcone C, laccino E, Tuccillo FM, Gaspari M, Trimboli $F$ et al. In vivo targeting and growth inhibition of the A20 murine B-cell lymphoma by an idiotype-specific peptide binder. Blood 2010; 116: 226-238.
13 Binder M, Muller F, Jackst A, Lechenne B, Pantic M, Bacher U et al. B-cell receptor epitope recognition correlates with the clinical course of chronic lymphocytic leukemia. Cancer 2011; 117: 1891-1900.

14 Seiler T, Woelfle M, Yancopoulos S, Catera R, Li W, Hatzi K et al. Characterization of structurally defined epitopes recognized by monoclonal antibodies produced by chronic lymphocytic leukemia B cells. Blood 2009; 114: 3615-3624.

This work is licensed under a Creative Commons AttributionNonCommercial-NoDerivs 4.0 International License. The images or other third party material in this article are included in the article's Creative Commons license, unless indicated otherwise in the credit line; if the material is not included under the Creative Commons license, users will need to obtain permission from the license holder to reproduce the material. To view a copy of this license, visit http:// creativecommons.org/licenses/by-nc-nd/4.0/
}

(c) The Author(s) 2016

\section{Targeted sequencing informs the evaluation of normal karyotype cytopenic patients for low-grade myelodysplastic syndrome}

\section{Leukemia (2016) 30, 2422-2426; doi:10.1038/leu.2016.247}

The diagnosis of myelodysplastic syndrome (MDS) requires persistent cytopenias, not otherwise explained, and evidence of morphologic dysplasia in the bone marrow. Low-grade MDS (bone marrow blasts $<5 \%$ ) has morphologic dysplasia in at least $10 \%$ of cells in one or more cell lineages. ${ }^{1}$ Low-grade MDS is particularly challenging to diagnose, as no definitive criteria for morphologic dysplasia exist and evaluation may be subject to high interobserver variability. ${ }^{1-3}$ The ability to diagnose low-grade MDS can be improved by incorporating cytogenetic evaluation of the bone marrow, especially in the setting of equivocal morphologic dysplasia. However, many MDS cases (up to 60\%) lack cytogenetic abnormalities, limiting the overall utility of cytogenetics as a diagnostic adjunct. ${ }^{4}$

Multiple studies have demonstrated that the majority of MDS patients ( $80 \%$ in some studies) harbor recurrent somatic mutations in a group of $20-30$ genes. $^{5-7}$ Further, some gene mutations confer an adverse prognosis independent of clinical scoring systems. $5,6,8$ We sought to determine whether targeted DNA sequencing of recurrently mutated MDS genes could be a useful adjunct in the diagnostically challenging subgroup of cytopenic patients with low blast counts and a normal karyotype, thereby identifying a subset of patients that may potentially be at a higher risk of developing MDS or acute myeloid leukemia (AML).

We screened 599 patients who presented between 1/2002 and $11 / 2015$, consented for sequencing studies on a protocol approved by the Human Research Protection Office at Washington University, and had banked bone marrow and control tissue (skin). Forty-three patients were selected based on (1) stringent cytopenia criteria (WBC $<1800 / \mu \mathrm{l}$, hemoglobin $<10 \mathrm{~g} / \mathrm{dl}$, platelets $<100 \mathrm{k} / \mu \mathrm{l}$ ) in at least one lineage, (2) bone marrow blasts $<5 \%$ by flow cytometry and/or morphologic evaluation (and had slides available for review), (3) WBC $<14 \mathrm{k} / \mu \mathrm{l}$, (4) nonclonal metaphase cytogenetics, and (5) absence of prior therapy for MDS (Table 1). Bone marrow specimens were independently reviewed (blinded) for blast count and dysplasia by two boardcertified hematopathologists (EJD and KV) and the percentage of dysplastic cells in the myeloid, erythroid and megakaryocytic lineages enumerated. Dysplasia was binned into categories of $<10,10-20,21-50$ and $>50 \%$. Definitive dysplasia was established when both pathologists identified dysplasia in $\geqslant 10 \%$ of cells in at least one lineage. Equivocal dysplasia was rendered when there was disagreement over the identification of $\geqslant 10 \%$ dysplasia in at least one lineage. No dysplasia was rendered when both pathologists agreed that dysplasia was $<10 \%$ in all lineages. Genomic DNA was extracted from bone marrow and skin (as a source of normal DNA) and enriched for the coding exons of a panel of 284 commonly mutated myeloid genes (Supplementary Table 1). ${ }^{5-8}$ DNA was extracted from aspirate coverslips for followup cases when cryopreserved cells were not available. Libraries were sequenced on a HiSeq 2500 (Illumina, San Diego, CA, USA) instrument with $2 \times 101 \mathrm{bp}$ reads. The resulting data was analyzed for single nucleotide variants (SNVs) and insertions/deletions (indels), using standard analysis pipelines in paired normal mode, as previously reported. ${ }^{9}$ To reduce false positive calls, only variants with $\geqslant 5$ variant reads, $\geqslant 50 \times$ total coverage in marrow and skin samples, $\geqslant 5 \%$ variant allele fraction (VAF, variant reads/total reads) in the marrow, not present in the database of short genetic variations (unless known canonical somatic hotspot mutations), and that resulted in protein coding changes were conservatively included in the analysis. Copy number alterations and loss-ofheterozygosity were called using the CopyCAT2 package.

Mean unique coverage depth was $265 \times$ for primary bone marrows, $252 \times$ for skin, and $388 \times$ for follow-up coverslips. Of the 43 sequenced cases, 29 had a coding-region somatic mutation in at least one gene (mean 2.8 mutations/case, range 1-8 mutations/ case). The most commonly mutated gene was SRSF2 (8 cases), followed by TET2 (7 cases), SF3B1 (6 cases) and U2AF1 (6 cases) (Figure 1a). Of the 284 sequenced genes, 40 were mutated in at 